ORIGINAL ARTICLE

\title{
Papillary carcinoma of the thyroid in Japan: subclassification of common type and identification of low risk group
}

\author{
K Kakudo, W Tang, Y Ito, I Mori, Y Nakamura, A Miyauchi
}

J Clin Pathol 2004;57:1041-1046. doi: 10.1136/jcp.2004.017889

See end of article for authors' affiliations

.....................

Correspondence to: Kennichi Kakudo, Department of Pathology, Wakayama Medical University, Kimiidera 8111, Wakayama, 641-8509, Japan; kakudo-k@ wakayama-med.ac.jp

Accepted for publication 15 April 2004
Aims: Papillary thyroid carcinoma (PTC) is classified into two subgroups-common type and other histological variants. Correlations between further subgrouping of the common type and patient prognosis are not well documented.

Aims: To introduce two novel histological parameters to characterise PTC-loss of cellular polarity and loss of cellular cohesiveness. To investigate a new subgroup of common type PTC with possible prognostic value.

Methods: In total, 213 patients with PTCs larger than $1 \mathrm{~cm}$ were studied. Histological characteristics of these PTCs, including tumour growth pattern, encapsulation, extrathyroidal invasion, loss of cellular polarity, and loss of cellular cohesiveness were examined and correlated with disease free survival (DFS). Results: Multivariate analysis revealed that invasive growth of unencapsulated PTC, in addition to sex (male) and tumour size $(>4 \mathrm{~cm}$ ) were significant and independent parameters for poor DFS, whereas loss of cellular polarity and cohesiveness, old age (>60 years), extrathyroid invasion, and completeness of surgery were significant only in univariate analysis. PTCs that showed expansive growth and retained cellular polarity had a favourable course, with no recurrence and no cancer related deaths. In contrast, PTCs exhibiting loss of cellular polarity and/or invasive growth with no tumour capsule had a higher risk of recurrence.

Conclusion: Cytological features alone cannot predict patient outcome in PTC. This study indicates for the first time that loss of cellular polarity and the tumour growth pattern are useful parameters for identifying the so called low risk group in common type PTC and in predicting patient outcome in terms of tumour recurrence and cancer related death.
$P$ apillary thyroid carcinoma (PTC) is a major histological type of differentiated carcinoma of the thyroid and most often has an excellent prognosis. PTC is diagnosed based on cytological characteristics, such as ground glass nuclei, intranuclear inclusions, or nuclear grooves. Histologically, PTC has been further subdivided into a common type and several other histological variants, including poorly differentiated PTC, ${ }^{1}$ insular carcinoma, ${ }^{2}$ tall cell variant, ${ }^{3}{ }^{4}$ and columnar cell variant..$^{5}$ In our previous study of PTC and parathyroid gland invasion, ${ }^{6}$ we were surprised to encounter only one cancer death and 22 recurrences in a series of 355 patients with PTC, most of who were classified as having common type PTC. This excellent prognosis was significantly different from that reported for malignant neoplasms derived from other human organs..$^{7-11}$ Therefore, we speculated that PTC of common type might include biologically benign or borderline lesions, which do not recur after simple surgical treatment. In our present study, we have identified the histological features of primary PTC of the thyroid that are associated with the extremely good survival that we reported previously. ${ }^{6}$ Characteristics examined in our study include loss of cellular polarity and loss of cellular cohesiveness, in addition to extrathyroid invasion, invasive growth patterns (presence or absence of tumour capsule), and poorly differentiated histological subtypes of PTCs (tall cell, columnar cell, and solid and trabecular growth patterns).

"In our present study, we have identified the histological features of primary PTC of the thyroid that are associated with the extremely good survival that we reported previously"

\section{MATERIALS AND METHODS}

Patients analysed in our study were surgically treated for PTC with or without lymph node dissection at Kuma Hospital, Kobe, Japan between February 1992 and June 1993. The 213 patients enrolled in our study had tumours larger than $1 \mathrm{~cm}$ in diameter and an average follow up period of 113 months (range, 60-139). They were treated with either total thyroidectomy (151 cases, including subtotal thyroidectomy) or lobectomy (114 cases, with or without isthmectomy). Table 1 summarises the clinical information from the 213 patients. The patients formed a consecutive series of primary papillary carcinomas of the thyroid and all histological subtypes or variants were included, such as encapsulated, follicular, diffuse sclerosing, tall cell, columnar cell, and poorly differentiated thyroid carcinoma, except for microcarcinoma. Other histological types of thyroid carcinoma such as follicular carcinoma, medullary (C-cell) carcinoma, anaplastic carcinoma, and malignant lymphomas were excluded from our study.

Sections of the PTCs were histologically evaluated by two pathologists (KK and WT). The histological parameters applied in our study-namely, loss of cellular polarity and loss of cellular cohesiveness-have not, according to previously published literature, been widely used for the characterisation of PTC. These parameters were first described by Tang et al to be associated with cell growth in PTC, indicated by MIB-1 labelling index and loss of retinoid receptor expression, an important receptor for morphogenesis

Abbreviations: DFS, disease free survival; PTC, papillary thyroid carcinoma 


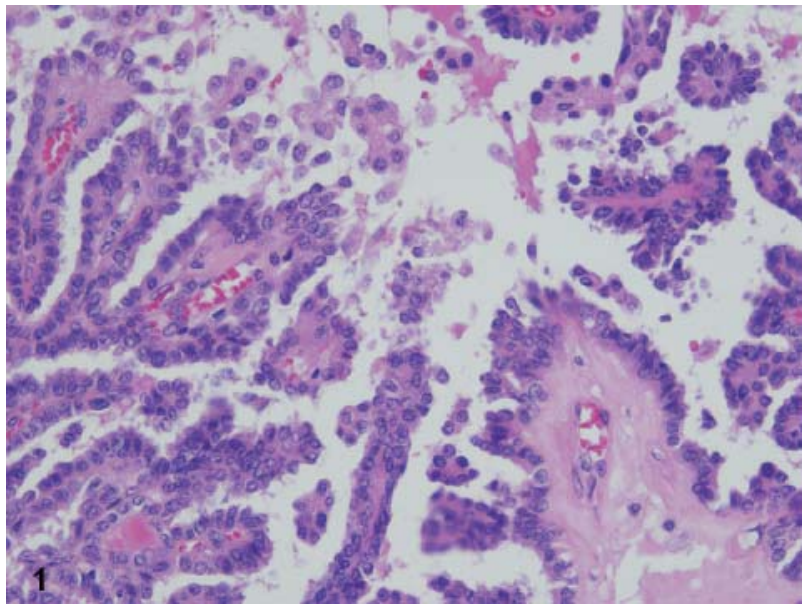

Figure 1 Group 2 papillary carcinoma of the thyroid: micropapillary structures with thin fibrovascular stroma are shown. They are covered with small, low cuboidal, or flattened epithelium with an increased nuclear to cytoplasmic ratio. Nuclear stratification along with cellular dissociation gives the epithelium a hobnail appearance. Haematoxylin and eosin stain; original magnification, $\times 20$.

and cell growth control. ${ }^{12}$ The features used to identify loss of cellular polarity in our study were as follows: presence of the nuclei in the middle or on top of the cytoplasm in cancer epithelium, analogous to a hobnail appearance (fig 1); raised nuclear position in tall columnar cancer cells; or irregular tubular or papillary structures covered with low, flattened, or small round cells without colloid substance (fig 2). In most cases, features of loss of cellular polarity could be identified, but the proportion of the tumour area showing these features varied among cases. In our previous study, a high correlation between loss of polarity and high MIB-1 labelling index was seen when the cutoff for the loss of polarity was set at representative features in $20 \%$ of the tumour area. ${ }^{12}$ The feature used to identify loss of cellular cohesiveness was

Table 1 Clinical information for 213 patients with papillary thyroid carcinoma larger than $1 \mathrm{~cm}$ in diameter

\begin{tabular}{|c|c|}
\hline \multicolumn{2}{|l|}{ Parameter } \\
\hline Age (mean) & 48.3 years \\
\hline \multicolumn{2}{|l|}{ Sex } \\
\hline $\begin{array}{l}\text { Male } \\
\text { Female }\end{array}$ & $10(4.7 \%)$ \\
\hline \multirow{2}{*}{\multicolumn{2}{|c|}{ Tumour size }} \\
\hline & \\
\hline$>4 \mathrm{~cm}$ & $28(13.2 \%)$ \\
\hline \multicolumn{2}{|c|}{ Extrathyroid invasion } \\
\hline Absent & $96(45.1 \%)$ \\
\hline Present & 117 (54.9\%) \\
\hline \multicolumn{2}{|c|}{ Nodal metastasis* } \\
\hline Negative & 41 (19.2\%) \\
\hline Positive & 160 (75.1\%) \\
\hline Unknown & $12(5.7 \%)$ \\
\hline Recurrence† & $20(9.4 \%)$ \\
\hline \multicolumn{2}{|l|}{ Stage } \\
\hline 1 & $82(38.5 \%)$ \\
\hline$\|$ & $16(7.5 \%)$ \\
\hline III & $103(48.4 \%)$ \\
\hline IV & \\
\hline Unknown & $12(5.7 \%)$ \\
\hline \multicolumn{2}{|c|}{$\begin{array}{l}\text { Staging was performed according to the TNM classification of the UICC, } \\
1997 . \\
\text { *Nodal status was not examined in } 12 \text { patients, and thus staging could } \\
\text { not be performed in these cases. †Recurrences occurred in lymph nodes } \\
\text { only (12), residual thyroid (1), skin (2), lymph node and lung (2), lung } \\
\text { only (1), lymph node and muscle (1), and lymph node, lung, and brain } \\
\text { (1) (total 20). }\end{array}$} \\
\hline
\end{tabular}

loosely or individually arranged cancer cells without papillary or follicular arrangement (often seen in the periphery of tumours) (fig 3). When loss of cellular cohesiveness was observed histologically in more than two areas (foci) in the tumour, the tumour was classified as positive for this parameter. Furthermore, the PTCs were histologically divided into two different growth pattern categories; namely, expansive growth and invasive growth. Tumours with expansive growth exhibited a sharp margin between the tumour tissue and the surrounding thyroid parenchyma, accompanied in some cases by a complete or incomplete capsule. Those tumours showing invasive growth infiltrated the thyroid parenchyma and lacked a sharp tumour margin or capsule.

\section{Tumour grouping}

Three parameters, namely_growth pattern, encapsulation, and loss of polarity-were used for PTC subgrouping after having been identified as significant prognostic factors by univariate analysis. Recurrences at any site after surgery were seen in PTCs with loss of polarity and those without loss of polarity but with invasive growth and no capsule. Therefore, tumours were assigned to group 1 if they exhibited expansive growth, with a complete or incomplete capsule, and if they showed loss of polarity in no more than $20 \%$ of the tumour area (fig 4). Group 2 PTCs included those tumours exhibiting loss of cellular polarity in more than $20 \%$ of the tumour area and/or an invasive growth pattern with no tumour capsule. This group included some tumours showing poorly differentiated histological patterns (tall cell, columnar cell, and solid and trabecular growth patterns) in a small part of their tumour area. Those tumours with predominantly poorly differentiated histological patterns as specified above were assigned to group 3 .

\section{Evaluation of recurrence}

Tumour recurrence was determined clinically using neck ultrasonography and serum thyroglobulin concentrations, in addition to routine physical examination. When palpable tumours were identified, they were examined cytologically. Of the 20 recurrent cases diagnosed cytologically, 18 patients underwent surgical treatment, and PTC was then confirmed histologically. The remaining two patients were cytologically

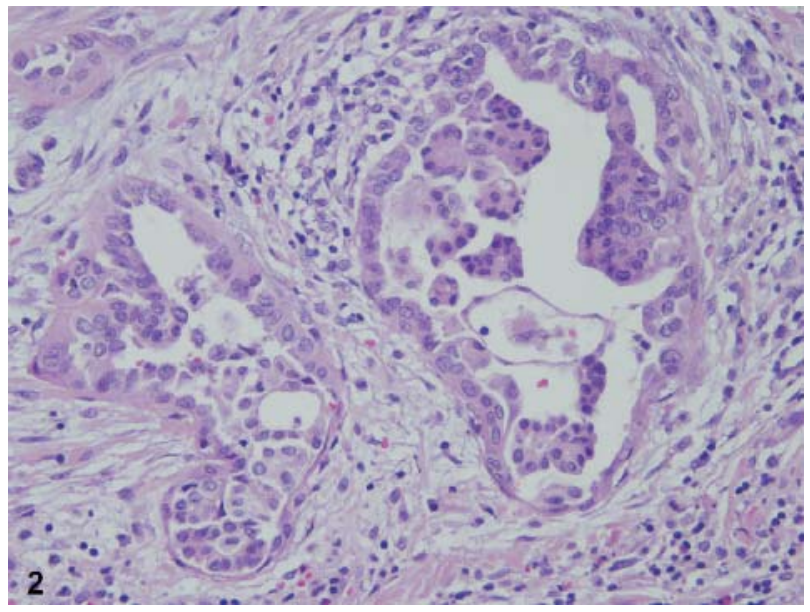

Figure 2 Irregular tubular and micropapillary structures covered with low cuboidal epithelium in group 2 papillary thyroid carcinoma. The epithelial cells are mostly small with an increased nuclear to cytoplasmic ratio. Note the absence of colloidal substances in the lumen. The presence of tubular spaces separates the histology seen here from the schirrous growth reported in so called poorly differentiated carcinomas. Haematoxylin and eosin stain; original magnification, $\times 20$. 


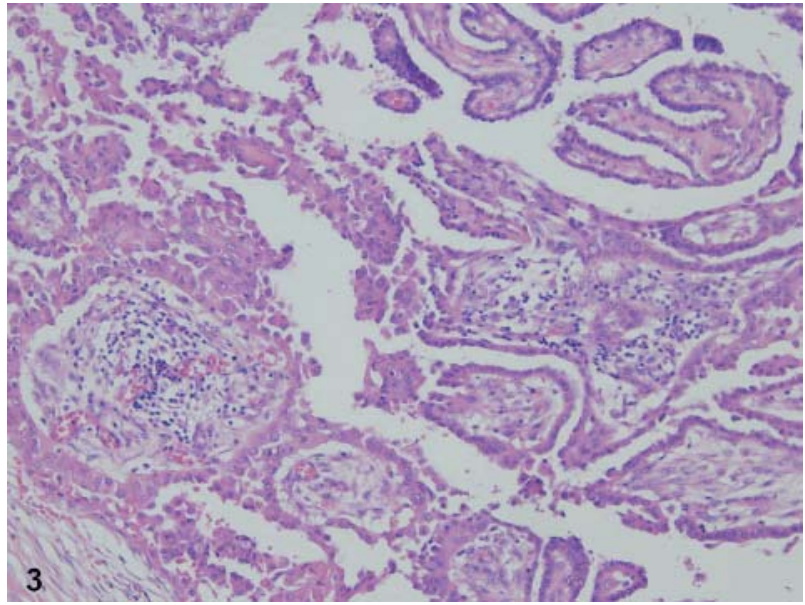

Figure 3 An invasive front of papillary thyroid carcinoma in a group 2 specimen, which contains groups of dissociated cells with hyperchromatic, round nuclei in their cytoplasm. Irregular micropapillary structures and piles of cancer nests are good examples of loss of polarity in group 2 papillary carcinoma. Haematoxylin and eosin stain; original magnification, $\times 10$.

diagnosed with PTC but had not received surgical treatment by the date of analysis (November 2003).

\section{Statistical analysis}

Time independent, categorical data were evaluated using the $\chi^{2}$ test or Student's $t$ test. Disease free survival (DFS) analysis was performed using Stat View-J, version 5.0 statistical software (SAS Institute Inc, Cary, New Carolina, USA). For univariate analysis of time dependent variables, the KaplanMeier method and the log rank test were used to determine prognostic factors. Univariate analysis was performed on the following parameters: age, sex, type of operation, tumour size, multiplicity, nodal metastasis, extrathyroid extension, encapsulation, surgical cut margin, cellular polarity, and cellular dissociation. All factors found to be significant by univariate analysis were subjected to multivariate analysis using Cox proportional hazards regression model. The

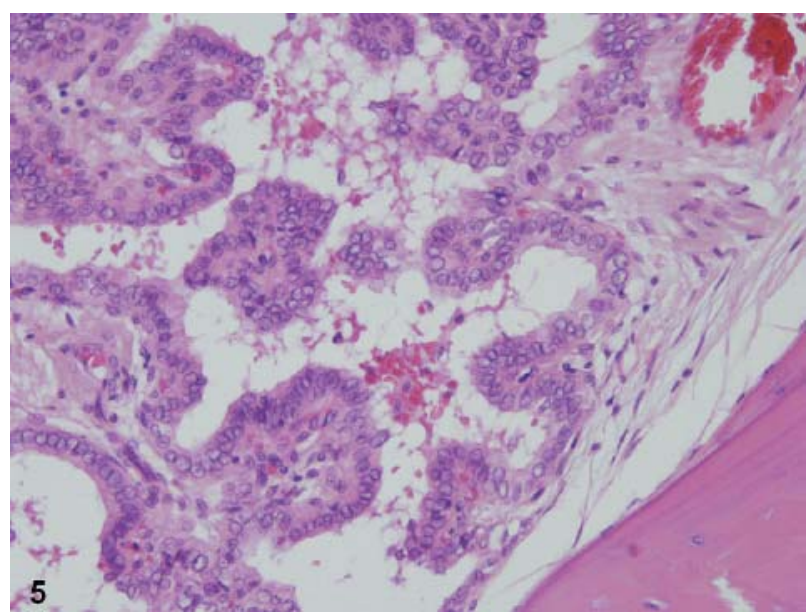

Figure 4 Papillary carcinoma of the thyroid in a group 1 specimen: papillary structures are covered with tightly attached cuboidal to columnar epithelial cells, which contain a large amount of cytoplasm and ground glass nuclei. Bone formation is also demonstrated in the stroma (lower right). Haematoxylin and eosin stain; original magnification, $\times 20$. differences were considered significant when the probability was less than 0.05 .

\section{RESULTS}

Recurrences were identified in 20 (9.4\%) of the 213 patients with PTC. There were 16 patients with lymph node recurrence, among whom, two also had lung metastases, one had perithyroid muscle metastasis, and one had metastases in both lung and brain. The remaining four recurrences included two cases in the cervical skin, one in the residual thyroid, and one in the lungs only. Only one cancer related death was identified in the 213 patients with PTC. The patient was a 56 year old woman, who on first presentation had a $4.5 \times 2.6 \mathrm{~cm}$ thyroid mass and palpable nodes. She was diagnosed as having a stage III PTC and was assigned to group 2 of our study. Five years after total thyroidectomy, she underwent surgery for nodal recurrence. However, she developed lung metastasis and finally succumbed to the disease ( 10 years after her initial operation).

Loss of cellular polarity was identified in 103 PTCs including 16 cases with recurrence. Univariate analysis demonstrated that the loss of cellular polarity was significantly correlated with poor DFS ( $p=0.0036)$ (table 2 ). An invasive growth pattern was seen in 92 PTCs, including 15 cases with recurrence. The patients with invasively growing PTCs also had a significantly poorer DFS compared with that of those patients with PTCs that exhibited expansive growth $(p=0.0033)$. Other factors correlated to poor DFS were age (60 years and over, $p=0.0055)$, male sex $(p=0.0119)$, tumour size $(>4 \mathrm{~cm}, \mathrm{p}<0.0001)$, positive extrathyroid extension ( $p=0.0002)$, and loss of cellular cohesiveness $(\mathrm{p}=0.0017)$. Multivariate analysis showed that invasive growth, sex, and tumour size were independent predictive factors of poor DFS (table 3 ).

In our study, the 213 PTCs were subclassified into three groups: group 1 with 67 cases $(31.4 \%)$, group 2 with 121 cases $(56.8 \%)$, and group 3 with 25 cases $(11.8 \%)$, based on tumour growth patterns, the extent of cellular polarity loss, and the presence of poorly differentiated histological features. Comparison of these three groups of patients (table 4) showed significant differences in extrathyroid invasion $(p=0.0003)$ and recurrence $(p=0.0034)$. However, no significant differences were seen between the groups with regard to patient age, sex, tumour size, nodal status, or stage distribution. Recurrence at any site after surgical treatment was found in none of the 67 group 1 patients, 17 of 121 of the group 2 patients ( $14.0 \%$ ), and three of the 25 group 3 patients $(12 \%)$. The 10 year DFS rate in group 1 was $100 \%, 85.5 \%$ in group 2, and $86.9 \%$ in group 3 (fig 5). However, comparison of DFS between groups 2 and 3 yielded no significant results.

Table 2 Univariate analysis of clinicopathological parameters of 213 papillary thyroid carcinomas

\begin{tabular}{lrl}
\hline Parameters & $\chi^{2}$ Value & p Value \\
\hline Age $(\leqslant 60 v>60$ years) & 7.691 & $0.0055^{*}$ \\
Sex (male $v$ female) & 6.319 & $0.0119^{*}$ \\
Diameter $(\leqslant 4 v>4 \mathrm{~cm}$ ) & 21.479 & $<0.0001^{*}$ \\
Multiplicity (multiple $v$ single) & 0.258 & 0.6113 \\
Extrathyroid invasion (absent $v$ present) & 17.032 & $0.0002^{*}$ \\
Growth pattern (expansive $v$ invasive) & 8.621 & $0.0033^{*}$ \\
Surgical cut margin (negative $v$ positive) & 3.142 & 0.0763 \\
Cellular polarity (retained $v$ lost) & 8.407 & $0.0036^{*}$ \\
Cellular dissociation (absent $v$ present) & 7.704 & $0.0055^{*}$ \\
\hline
\end{tabular}

Univariate analyses were performed using the Kaplan-Meier method, and the $\chi^{2}$ and $p$ values were obtained using the log rank (Mantel-Cox) test ( ${ }^{*} \mathrm{p}<0.05$, significant). 
Table 3 Multivariate analysis of clinicopathological parameters of 213 papillary thyroid carcinomas

\begin{tabular}{lllll}
\hline Parameters & RC & SE & OR (95\% Cl) & p Value \\
\hline Age ( $\geqslant 60$ years) & -0.761 & 0.494 & $0.467(0.177$ to 1.231$)$ & 0.1236 \\
Sex (male) & -1.487 & 0.744 & $0.226(0.053$ to 0.971) & $0.0455^{*}$ \\
Diameter ( $>4 \mathrm{~cm})$ & -1.672 & 0.596 & $0.188(0.058$ to 0.805$)$ & $0.0051^{*}$ \\
Extrathyroid invasion & -0.556 & 0.916 & $0.574(0.095$ to 3.456$)$ & 0.5439 \\
Loss of polarity & -1.186 & 0.619 & $0.305(0.091$ to 1.028$)$ & 0.0554 \\
Loss of cohesiveness & -0.425 & 0.585 & $0.654(0.208$ to 2.056$)$ & 0.4669 \\
Invasive growth pattern & -1.130 & 0.563 & $0.323(0.107$ to 0.974$)$ & $0.0448^{*}$ \\
\hline *Significant. & \multicolumn{7}{l}{} \\
Cl, confidence interval; OR, odds ratio; RC, regression coefficient; SE, standard error. &
\end{tabular}

\section{DISCUSSION}

PTCs are diagnosed on the basis of their cytological features. However, those histological characteristics that are useful for predicting patient outcome are ill defined. Therefore, histological characteristics such as encapsulation, invasive growth, and lymph node metastasis are not currently regarded as essential for the diagnosis or prognosis of PTC. In this paper, we have identified three prognostically different groups of PTC based on simple histological parameters; namely, loss of cellular polarity, invasive growth pattern, and poorly differentiated histological characteristics. Analyses of patient outcomes in the three groups revealed that the tumours fell into the following categories: (1) low risk group (biologically benign), in which patients develop no recurrences or metastases after surgery and in which no cancer related deaths occur; (2) low grade malignant tumours (common type), where recurrences occur in approximately $10 \%$ of patients after surgical treatment; and (3) poorly differentiated tumours, as previously defined..$^{1-5}{ }^{13}{ }^{14}$ Shaha et al in 1996 and 1997 reported three different risk groups in their 810 cases of PTC. ${ }^{15}{ }^{16}$ Parameters associated with low risk PTC were the following: patient age (younger than 45 years); tumour size $(<4 \mathrm{~cm}$ in diameter); low histological grade; absence of distant metastasis; and absence of extrathyroidal extension. Of the 810 cases, 403 were classified as low risk, 313 as intermediate risk, and 93 as high risk, with 20 year survival rates of $99 \%, 83 \%$, and $43 \%$, respectively. ${ }^{15}$ Of the cases of well differentiated thyroid carcinoma identified in by Shaha et al, $44.8 \%$ were classified as low risk and $38.6 \%$ as intermediate risk compared with our study, in which group 1 represented $31.4 \%$ and group 2 $56.8 \%$ of the patients with well differentiated carcinoma. Even though different study parameters were applied, purely histological in our study compared with histological and clinical in the study of Shaha et al, it is possible that our

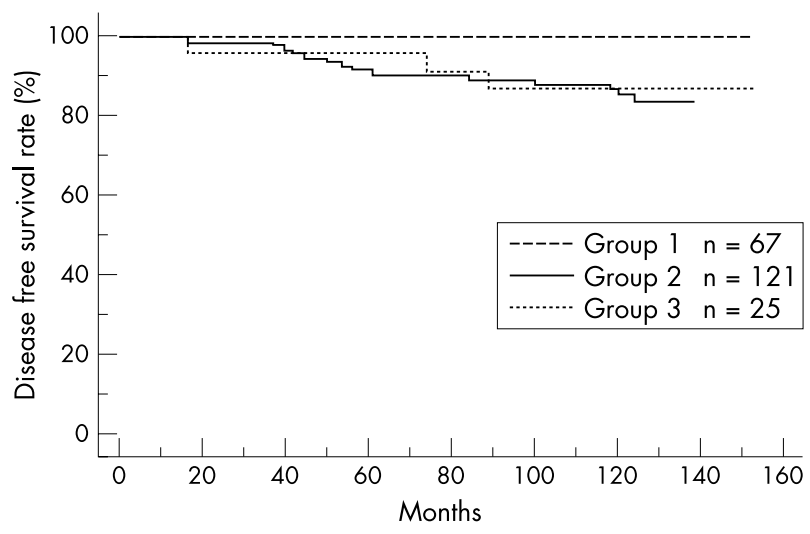

Figure 5 Histological grouping of papillary thyroid carcinomas and disease free survival (Kaplan-Meier). group 1 (biologically benign low risk group) and group 2 (low grade malignant common type) correspond to the low risk and intermediate risk groups, respectively, identified by Shaha et al.

We report for the first time that loss of cellular polarity is a significant histological indicator of the risk of developing recurrence in PTC. This histological parameter has not been described previously in studies covering usual type, common type, aggressive type, or poorly differentiated PTCs. We believe that the loss of cellular polarity may be a phenotypic expression and histological characteristic of poor cellular differentiation. The loss of cellular polarity may provide a better indication of the risk of recurrence and may also be a simpler parameter than other prognostic indicators currently used. ${ }^{15-21}$ However, our illustrations of loss of cellular polarity and loss of cohesiveness (figs 1-3) do not match previously reported poorly differentiated histological characteristics, although these characteristics may have been seen in the usual type or common type of well differentiated papillary carcinoma. The elevated nuclear position and the nuclear position seen in hobnail cancer epithelium may both be linked to the loss of nuclear polarity, which may, in addition, be linked to the tall cell variant, with basally located nuclei, and the columnar cell variant with stratified nuclei in a wide cytoplasm. The small cancer cells with a high nuclear to cytoplasmic ratio and the flat epithelial cells with little cytoplasm, which cover irregular tubular and micropapillary structures, have not been described as other variants of poorly differentiated PTC, but it may be that the characteristics of these cells indicate the loss of cellular function or cellular differentiation to a lesser degree.

\section{"We believe that the loss of cellular polarity may be a phenotypic expression and histological characteristic of poor cellular differentiation"}

To the best of our knowledge, there is no general agreement regarding how best to separate PTCs based on histopathological characteristics into prognostically different subgroups. ${ }^{19}$ Sakamoto et al reported that the five year cumulative survival rate was $65 \%$ for the $15.6 \%$ of PTCs that were poorly differentiated, whereas patients with well differentiated thyroid carcinoma had a $95.1 \%$ survival rate. ${ }^{1}$ They also pointed out that non-glandular components (solid, trabecular, and schirrous) are characteristics of poorly differentiated PTCs, which are likely to recur within five years of the first excision. They did not describe the nonglandular histology in terms of poor cellular differentiation or loss of cellular differentiation, but described the clinicopathological condition as poorly differentiated carcinoma. We believe that the term poorly differentiated carcinoma should be assigned to cases that develop early recurrence leading to high death rates, as described by Sakamoto et al. According to 


\begin{tabular}{|c|c|c|c|c|}
\hline & $\begin{array}{l}\text { Group } 1 \\
(n=67)\end{array}$ & $\begin{array}{l}\text { Group 2 } \\
(n=121)\end{array}$ & $\begin{array}{l}\text { Group } 3 \\
(n=25)\end{array}$ & $\mathrm{p}$ Value \\
\hline Age (average) & 46.3 & 49.6 & 47.9 & NS \\
\hline \multicolumn{5}{|l|}{ Sex } \\
\hline M & 2 & 8 & 0 & \multirow{2}{*}{ NS } \\
\hline $\mathrm{F}$ & 65 & 113 & 25 & \\
\hline \multicolumn{5}{|l|}{ Tumour size } \\
\hline $1-4 \mathrm{~cm}$ & 62 & 102 & 21 & \multirow[t]{2}{*}{ NS } \\
\hline$>4 \mathrm{~cm}$ & 5 & 19 & 4 & \\
\hline \multicolumn{5}{|c|}{ Extrathyroid invasion } \\
\hline$(-)$ & 43 & 40 & 13 & \multirow{2}{*}{$\begin{array}{l}0.0002 \\
(1 \vee 2 \vee 3)^{*}\end{array}$} \\
\hline$(+1)$ & 24 & 81 & 12 & \\
\hline \multicolumn{5}{|c|}{ Nodal metastasis } \\
\hline Negative & 17 & 19 & 5 & \multirow[t]{3}{*}{ NS } \\
\hline Positive & 43 & 99 & 18 & \\
\hline Unknown & 7 & 3 & 2 & \\
\hline Recurrence & $\begin{array}{l}0 / 67 \\
(0 \%)\end{array}$ & $\begin{array}{r}17 / 121 \\
(14 \%)\end{array}$ & $\begin{array}{l}3 / 25 \\
(12 \%)\end{array}$ & $\begin{array}{l}0.0060 \\
(1 \vee 2 \vee 3) \dagger\end{array}$ \\
\hline \multicolumn{5}{|l|}{ Stage } \\
\hline 1 & 29 & 43 & 10 & \multirow[t]{5}{*}{ NS } \\
\hline$\|$ & 7 & 7 & 2 & \\
\hline III & 24 & 68 & 11 & \\
\hline IV & 0 & 0 & 0 & \\
\hline Unknown & 7 & 3 & 2 & \\
\hline \multicolumn{5}{|c|}{$\begin{array}{l}\text { The } p \text { values were obtained using the } \chi^{2} \text { test or Student's } t \text { test }(p<0.05 \text { was considered significant). } \\
\text { *The } p \text { values were: }<0.0001 \text { (group } 1 \vee \text { group 2), NS (group } 1 \vee \text { group 3), and NS (group } 2 v \text { group 3); the } p \\
\text { values were } 0.0005 \text { (group } 1 \vee \text { group 2), } 0.0039 \text { (group } 1 \vee \text { group 3), and NS (group } 2 v \text { group 3). } \\
\text { NS, not significant. }\end{array}$} \\
\hline
\end{tabular}

this definition, PTCs in group 3 of our study are not classed as poorly differentiated carcinomas. We had a total of 25 cases of PTC with poor cellular differentiation in our present study when a cutoff of $>20 \%$ of the tumour area was applied, but these cases did not show a high incidence of nodal recurrence or other aggressive behaviour. DFS did not differ between groups 2 and 3, and thus these groups could be combined as far as DFS is concerned. We also believe that the tumours in group 2 of our study were of low grade malignancy and should not be called poorly differentiated carcinoma or aggressive type PTC. The invasive growth pattern is an important prognostic parameter in many other cancers but has been neglected thus far in the diagnosis of thyroid cancer; our paper is the first to point out its biological significance in PTC. Similar to previous reports, age and sex correlated with DFS, but they were not included in our histological grouping of PTC as clinical factors.

In a study by Akslen and LiVolsi, 128 cases of PTC were classified into three groups: usual type, complex type, and specific variant subtype, based on histological subtypes, identification of variants, and histological grading (pronounced nuclear atypia, tumour necrosis, and vascular invasion). ${ }^{19}$ Fifty five per cent of their PTC cases were of the usual type, corresponding most likely to the group 1

\section{Take home messages}

- Cytological features alone cannot predict patient outcome in papillary thyroid carcinoma (PTC)

- We report for the first time that loss of cellular polarity and the tumour growth pattern are useful parameters for identifying the so called low risk group in common type PTC

- These parameters should help predict patient outcome in terms of tumour recurrence and cancer related death and prevent overtreatment of low risk patients
(31.4\%) and group $2(56.8 \%)$ cases in our study. We propose that the common type of PTC should be subclassified into two groups: one group whose members have a high recurrence risk and will need close follow up (group 2); and one group in which tumours rarely recur and, whose members therefore require less aggressive treatment (group 1).

Williams et al in 2000 proposed the new term well differentiated tumour of uncertain malignant potential, which was to be applied only to encapsulated PTCs with minor nuclear changes. ${ }^{22}$ This concept suggests that there are PTCs with minor nuclear changes, which demonstrate "nonmalignant" potential. Although this term cannot be applied to tumours in group 1 of our study, because these tumours were malignant, with nodal metastasis in approximately $70 \%$ of the cases at the time of surgery, most group l patients were successfully cured by a surgical procedure analogous to that conducted for benign adenomas. Encapsulated papillary lesions with all the histological features of PTC were formerly defined as papillary adenoma, but are presently called encapsulated PTC. $^{23}$ We believe that the term papillary adenoma is not suitable to describe the group I PTCs in our study because of the high incidence of nodal metastasis in this group.

Traditional PTC diagnosis is based strictly on nuclear characteristics, regardless of histological characteristics of invasiveness, cellular differentiation, or even metastasis. The histological criteria applied in our study to define groups 1 and 2 may be helpful in identifying cases of PTC that are unlikely to recur or lead to cancer related death. We believe that the identification of this new group of PTC, described as the low risk group here, will help medical practitioners to avoid overtreating such patients.

\section{ACKNOWLEDGEMENTS}

The authors wish to express their gratitude to J A Carney (Emeritus staff of Mayo Clinic) and Professor JR Goellner, Mayo Clinic, for their valuable advice and discussions; Ms S Garvin for secretarial help; and Mr S Morita, Kuma Hospital, Kobe City, for his help with tissue preparation. 


\section{Authors' affiliations}

K Kakudo, W Tang, I Mori, Y Nakamura, Department of Pathology, Wakayama Medical University, Wakayama, 641-8509, Japan

Y Ito, A Miyauchi, Kuma Hospital, Kobe, 650-0011, Japan

\section{REFERENCES}

1 Sakamoto A, Kasai N, Sugano H. Poorly differentiated carcinoma of the thyroid. A clinicopathologic entity for a high-risk group of papillary and follicular carcinomas. Cancer 1983;52:1849-55.

2 Carcangiu ML, Zampi G, Rosai J. Poorly differentiated ("insular") thyroid carcinoma. A reinterpretation of Langhans" "wuchernde struma". Am J Surg Pathol 1984;8:655-8.

3 Hawk WA, Hazard JB. The many appearances of papillary carcinoma of the thyroid. Comparison with common form of papillary carcinoma by DNA and morphometric analysis. Cleve Clin Q 1976:43:207-16.

4 Johnson TL, Lloyd RV, Thompson NW, et al. Prognostic implications of the tall cell variant of papillary thyroid carcinoma. Am J Surg Pathol 1988;12:22-7.

5 Evans HL. Columnar-cell carcinoma of the thyroid. A report of two cases of an aggressive variant of thyroid carcinoma. Am J Clin Pathol 1986;85:77-80.

6 Kakudo K, Tang W, Ito Y, et al. Parathyroid invasion, nodal recurrence, and lung metastasis by papillary carcinoma of the thyroid. J Clin Pathol 2004:57:245-9.

7 Nzeako UC, Goodman ZD, Ishak KG. Hepatocellular carcinoma in cirrhotic and noncirrhotic livers. A clinico-histopathologic study of 804 North American patients. Am J Clin Pathol 1996;105:65-75.

8 Enblad P, Adami HO, Bergstrom R, et al. Improved survival of patients with cancers of the colon and rectum? J Natl Cancer Inst 1988 15;80:586-91.

9 Pescatori M, Mattana C, Maria G, et al. Outcome of colorectal cancer. Br J Surg 1987;74:370-2.

10 Nakamura K, Ueyama T, Yao T, et al. Pathology and prognosis of gastric carcinoma. Findings in 10,000 patients who underwent primary gastrectomy. Cancer 1992 1;70:1030-7.

11 Soga J, Yakuwa Y. Bronchopulmonary carcinoids: an analysis of 1,875 reported cases with special reference to a comparison between typical carcinoids and atypical varieties. Ann Thorac Cardiovasc Surg 1999;5:211-19.

12 Tang W, Nakamura Y, Zuo H, et al. Differentiation, proliferation and retinoid receptor status of papillary carcinoma of the thyroid. Pathol Int 2003:53:204-13.

13 Pilotti S, Collini P, Manzari A, et al. Poorly differentiated forms of papillary carcinoma: distinctive entities or morphological patterns? Semin Diagn Pathol 1995; 12:249-55

14 Sobrinho-Simoes $M$, Sambade C, Fonseca E, et al. Poorly differentiated carcinoma of the thyroid gland. A review of the clinicopathologic features of a series of 28 cases of heterogenous clinically aggressive group of thyroid tumors. Int J Surg Pathol 2002;10:123-31.

15 Shaha AR, Shah JP, Loree TR. Risk-group stratification and prognostic factors in papillary carcinoma of thyroid. Ann Surg Oncol 1996:3:534-8.

16 Shaha AR, Shah JP, Loree TR. Low-risk differentiated thyroid cancer. The need for selective treatment. Ann Surg Oncol 1997;4:328-33.

17 Shaha AR, Shah JP, Loree TR. Patterns of nodal and distant metastasis based on histologic varieties in differentiated carcinoma of the thyroid. Am J Surg 1996;172:692-4.

18 Akslen LA. Prognostic importance of histologic grading in papillary carcinoma. Cancer 1993;72:2680-5.

19 Akslen LA, LiVolsi VA. Prognostic significance of histological grading compared with subclassification of papillary carcinoma. Cancer 2000;88:1902-8.

20 Hay ID, Bergstralh EJ, Goellner JR, et al. Predicting outcome in papillary carcinoma: development of a reliable prognostic scoring system in a cohort of 1,779 patients surgically treated at one institution during 1940 through 1989. Surgery 1993;114:1050-7.

21 Wada N, Duh QY, Sugino K, et al. Lymph node metastasis from 259 papillary carcinomas: frequency, pattern of occurrence and recurrence, and optimal strategy for neck dissection. Ann Surg 2003;237:399-407.

22 Williams ED, Abrosimov A, Bogdanova T, et al. Guest editorial: two proposals regarding the terminology of thyroid tumors. Int J Surg Pathol 2000;8:181-3.

23 Rosai J, Carcangiu ML, DeLellis RA, eds. Atlas of tumor pathology. Tumors of the thyroid gland, Series 3, Fascicle 5. Washington DC: Armed Forces Institute of Pathology, 1992.

\section{$\mathrm{ECHO}$}

\section{Antibodies against Saccharomyces cerevisiae and early surgery for Crohn's disease}

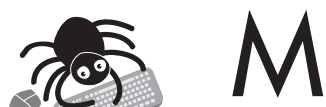

edical therapies for Crohn's disease (CD) range from the relatively benign and less effective (5-aminosalicylates and antibiotics) to the more toxic but more effective (steroids, immunomodulators, and anti-tumour necrosis factor antibodies). Despite such treatments about $80 \%$ of patients need surgery within 20 years of diagnosis and 20 $40 \%$ within three years. Earlier more aggressive medical treatment might reduce rates of Please visit the Journal of Clinical Pathology website [www. jclinpath.com for a link to the full text of this article. surgery but better methods of assessing prognosis are needed so that early effective but potentially toxic treatments can be reserved for patients who might benefit most. AntiSaccharomyces cerevisiae antibodies (ASCA) are found in $60 \%$ of patients with CD and are nearly $95 \%$ specific for the diagnosis. There is evidence that ASCA are indicative of poorer prognosis. Now researchers in New England, USA, have reported an association between ASCA and early need for surgery.

A cohort study at 16 centres included 345 patients with CD diagnosed between 1991 and 1999 and followed for at least three years. Sixty nine of these patients required major surgery for CD (excluding surgery at diagnosis) within three years of diagnosis. The case control study included 35 cases (major surgery within three years) and 35 controls (no major surgery within three years), 30 of them matched for age, sex, disease location, and smoking behaviour. Blood was taken for ASCA testing after surgery or, in controls, more than three years after diagnosis. ASCA tests were positive in $23 / 35$ cases $(66 \%)$ and $7 / 35$ controls (20\%). Positive IgA ASCA increased the risk of early surgery eightfold, positive IgG ASCA increased it 5.5-fold, and positive IgA and IgG ASCA increased it fivefold. The significant association between IgA ASCA and early surgery applied to ileocaecal resection and complex intra-abdominal abscess drainage but not to colon resection or complex perianal surgery. Measuring antibody titres did not add to the value of the results. Other markers of inflammatory bowel disease (perinuclear antineutrophil cytoplasmic antibodies and Escherichia coli outer membrane porin C were not associated with early surgery. ASCA titres were not related to time after surgery or time since diagnosis.

Patients with CD who have had surgery within three years of diagnosis are more likely to be ASCA positive than are patients who have not had surgery within three years of diagnosis. More work is needed to demonstrate whether ASCA status at diagnosis is predictive of more aggressive disease and whether more intensive treatment for ASCA positive patients would give better long term results.

A Forcione DG, et al. Gut 2004;53:1117-1122. 